Dijkerman, H.C., Smit, M.C.

Interference of grasping observation during prehension, a behavioural study.

Experimental Brain Research: 2006, nr. 8, p.

\begin{tabular}{|l|l|}
\hline Postprint Version & 1.0 \\
Journal website & $\underline{\text { http://www.springerlink.com/ }}$ \\
\hline Pubmed link & $\begin{array}{l}\text { http://www.ncbi.nlm.nih.gov/entrez/query.fcgi?db=pubmed\&cmd=Retrieve\&dop } \\
\text { t=AbstractPlus\&list uids=16917772\&query hl=14\&itool=pubmed docsum }\end{array}$ \\
\hline DOI & \begin{tabular}{l}
$10.1007 /$ s00221-006-0627-z \\
\hline
\end{tabular}
\end{tabular}

H. C. Dijkerman $(\triangle)$ - M. C. Smit

Helmholtz Research Institute, Utrecht University, Heidelberglaan 2, 3584 CS Utrecht, The Netherlands

e-mail: c.dijkerman@fss.uu.nl

\title{
Interference of grasping observation during prehension, a behavioural study
}

\author{
H. C. DIJKERMAN • M. C. SMIT
}

\begin{abstract}
During the last 10 years a considerable number of neurophysiological and functional imaging studies have provided evidence that observation and execution of movements activate common representations. Furthermore, several behavioural studies suggest that action observation can influence the performance of movements. Recently it was shown that viewing incongruent movements interferes with the execution of non-object oriented sinusoidal arm movements (Kilner et al. in Curr Biol 13:522-525, 2003). In the current study, we investigated whether interference of action observation also occurs during goaldirected prehension movements. Participants were required to grasp cubes of different sizes while simultaneously observing an actor performing grasping or pointing movements. The actors' movement could be directed at objects that were identical, or different in size to the cube grasped by the participant. The results showed that maximum grip aperture was affected by observation of grasping towards larger objects. No effect of object size was found during observation of pointing movements. These results suggest that observation of grasping movements can interfere with the on-line control of prehension movements and provides further evidence for overlapping networks for grasping observation and execution.
\end{abstract}

\section{INTRODUCTION}

In recent years, there has been increasing evidence to support the idea that action observation and execution activate common movement representations. Early electrophysiological studies with monkeys performed by Rizzolatti and colleagues (di Pellegrino et al. 1992; Gallese et al. 1996; Rizzolatti et al. 1996a) found a group of neurons in area F5 that not only discharged when performing certain hand movements, but also when observing them.

They further reported that a certain congruence can exist between motor and visual selectivity for these 'mirror neurons'. The degree of congruence mirror neurons display varies mutually. For some of the mirror neurons the congruence is strict, which means that not only the general action must match (for example, grasping), but also the way it is executed (for example, power grasp) (Gallese et al. 1996). For other mirror neurons the congruence can be broader. If this is the case, the motor requirements (for example, precision grip) are usually stricter than the visual requirements (for example, any type of hand grasping) (Rizzolatti and Arbib 1998; Fadiga et al. 2000). Typically, mirror neurons do not respond to the sight of a hand mimicking an action in the absence of the target. 
Similarly, they do not respond to the observation of an object alone, even when it is of interest of the monkey. To be triggered during observation, the mirror neurons require a particular interaction between another individual and an object. Even though conceptually identical to the original hand action, actions made by using tools, such as grasping with a pliers, barely activate mirror neurons (di Pellegrino et al. 1992; Gallese et al. 1996; Rizzolatti et al. 1996a; Fadiga et al. 2000).

Several studies have shown that action observation and motor performance also activate similar representations in humans. Fadiga et al. (1995) used transcranial magnetic stimulation (TMS) to show an increased excitability of the motor system when participants observed movements. This increase was found only in those muscles required when actively performing the observed movements. Rizzolatti et al. (1996b) used positron emission tomography (PET) to localize active brain regions during the observation of grasping movements. Grasping observation was related to a highly signifficant activity of the cortex of the middle temporal gyrus including that of the adjacent superior temporal sulcus (Brodmann's area 21) in the left hemisphere and the caudal part of the inferior frontal gyrus (Brodmann's area 45) in the left hemisphere. The latter being in line with suggestions of F5 being the monkey homolog of human Broca's area (Rizzolatti et al. 1996a). Broca's area was also reported by Iacoboni et al. (1999) who used functional magnetic resonance imaging (fMRI) to test whether imitation may be based on an action observation execution matching system. They found a significantly stronger activation in the left inferior frontal cortex (Broca's area), right anterior parietal region and right parietal operculum — during imitation than during execution of the same movement in response to spatial or symbolic cues. Nishitani and Hari (2000) attempted to reveal the relative timing of different areas of the mirror system, during execution, observation and imitation of hand movements by using a combination of MEG and MRI. Their findings suggested that the left BA 44, posterior Broca's area, acts as a sort of orchestrator of the human mirror system, strongly involved in action imitation.

However, for human imitation to be based on a mirror system homologous to the monkey mirror system, evidence is required that actions directed at targets are influenced by action observation. Indeed, as was mentioned earlier, in monkeys it takes object-oriented action for mirror neurons to become active. A recent behavioural study by Wohlschlager and Bekkering (2002) showed that human imitation is indeed driven by visual targets. Participants had to imitate downward movements of an index finger in a response time experiment. In one condition, the observed finger touched one of two dots on the surface, ipsilaterally or contralaterally. In the other condition, the observed finger again touched the surface ipsilaterally or contralaterally, but the two dots being absent. The presence of the dots had no effect on the movement proper, but did have a decisive influence on error patterns and response times. Dots specifically reduced the onset latency of ipsilateral finger movements and they specifically increased the use of the wrong finger when contralateral movements were required. These finding are supported by the results of an fMRI study in which the effect of explicit action goals on neuronal activity during imitation was assessed (Koski et al. 2002).

Considering that the first mirror neuron studies found that the majority of coactivity was between the observation and execution of visuomotor grasping, it is surprising that few studies have investigated the effects of grasping observation on performance of prehension in humans. Castiello et al. (2002) demonstrated that the observation of a human object-oriented hand action facilitated the subsequent execution of that hand action. The findings of Edwards et al. (2003) suggested that this effect occurred automatically. Thus, these studies show that observation prior to the performance of a visuomotor grasp can affect the preparation of this movement. Furthermore, Craighero et al. (2002) reported an influence in the opposite direction. They observed that motor preparation to grasp a bar facilitated the response to congruent visually presented hand pictures. However, these studies did not investigate whether observation of grasping objects influences an ongoing prehension movement.

Kilner et al. (2003) did observe an interference effect between observed and simultaneously executed movements. The movements made and observed in this study were not goal-directed, but rather consisted of sinusoidal arm movements. The current study investigated whether observing someone making a grasping movement interfered with the execution of an ongoing prehension movement. More specifically, we assessed whether the size of the observed grip aperture affected the hand opening during the grasping movement. Participants were asked to perform simple visuomotor grasping movements towards cubes, while simultaneously observing an actor performing prehension movements towards objects that were either smaller or larger than their own object. We hypothesized 
that observation of grasping a differently sized object would activate a representation of a different grasping movement which would lead to interference during execution of their own prehension movement.

\section{MATERIALS AND METHODS}

\section{Participants}

Five female and seven male subjects aged 20-33 years participated in this study. All participants were right handed and had normal, or corrected to normal, vision. The participants were naïve regarding the purpose of the study. Prior to participation in the study, participants gave informed consent. This study was performed in accordance with the principles laid down in the Helsinki Declaration and with the ethical advisory committee of the Faculty of Social Sciences of the Utrecht University, the Netherlands.

\section{Experimental set-up}

Two sets of three black cubes of were used measuring 100, 50 and $10 \mathrm{~mm}^{3}$, respectively. The experimenter used one set of cubes to grasp and point at. The other set of cubes was used by the participants to grasp. The cubes were made of solid black PVC and presented on a table against a white background.

A female 51-year-old right-handed experimenter, live performed the grasping and pointing movements that had to be observed. She also prepared each new trial by placing the required cubes on the table and removing the previous ones. For one participant, a different, female 22-year-old experimenter performed the movements. Since this participant's data did not differ from the rest of the data, it was included in the analyses.

Participants' grip aperture was measured by means of a magnetic recording system (Minibird, Ascension Technology). Markers were attached to the nails of participant's right index finger and thumb, measuring finger-thumb separation during the grasping movement, at a sampling rate of 86.1 Hz. The main dependent variable of interest was the maximum grip aperture (MGA) attained during reaching. In healthy participants, the amplitude of MGA covaries linearly with object size, providing a direct index of visual information usage prior to grasping an object (Jeannerod et al. 1995). In addition, grip aperture was calculated at 25, 50 and $75 \%$ of the movement (GA25, GA50, GA75) to assess during which part of the movement possible interference may occur. Several other kinematic variables were also extracted: movement time (MT), peak velocity (PV), time to peak velocity (TPV), time to peak velocity as percentage of total movement time (\%TPV), time to maximum grip aperture (TMGA) and time to maximum grip aperture as percentage of total movement time (\%TMGA). Reach related variables such as MT, PV, TPV and \%TPV were calculated using the data from the thumb marker.

\section{Design}

The participants performed grasping movements (grasping a cube) while observing other grasping movements that were either congruent (grasping an identical cube) or incongruent (grasping a larger or smaller cube). In addition, participants also observed grasping without a cube. This condition served as a baseline condition; it demanded the same amount of attention from the participant but, because of the absence of a graspable object, it did not represent a genuine grasping movement. Therefore, it should not lead to the same interference as an observed grasp would.

To ascertain that a possible interference effect is not due solely to the observation of a second differing cube on the table, another four conditions were added. In these conditions, the grasping movements made by the experimenter were replaced by pointing movements. Other than that, the pointing conditions were identical to the grasping conditions. Observing the pointing movements demanded the same amount of attention from the participant as observing the grasping movements. If a possible interference effect extends to the pointing conditions, it cannot be linked to grasp observation, but rather to observation of the object (size) itself.

The data were analysed only from those conditions in which the medium cube was grasped by the participant. Grasping of the large cube and the small cube by the participant was included for distracter purposes only. Thus, the experiment consisted of 16 different conditions, 8 test conditions (4 grasping and 4 pointing conditions) and 8 conditions that were not included in the analysis (see Table 
1). The test conditions were each presented ten times, the non-analysis conditions four times. These 112 trials were presented in 2 blocks of 56 trials. The order of presentation of the conditions was pseudo-randomized within the blocks.

\section{[ TABLE 1 ]}

\section{Movements made by the experimenter}

The stimuli consisted of the experimenter performing four grasping movements (see Fig. 1, top) and four pointing movements (see Fig. 1, bottom). In the grasping conditions, the experimenter grasped the cube without lifting it from its place. In the grasping condition without a cube, the experimenter simulated the grasping of a medium cube by 'grasping' in the direction of the spot on the table where in other conditions the cube was placed, and touching this spot with all fingers of her 'grasping' hand. In the pointing conditions involving cubes, the experimenter pointed at the cube by touching the centre of the top of the cube with her index finger. In the pointing condition without a cube, the experimenter pointed at the spot on the table where in the other conditions the cube was placed and touched it with her index finger. In the pointing conditions, the experimenter touched the cubes to resemble the interaction with the object during the grasping movement.

\section{[ FIGURE 1 ]}

The experimenter and participant were sitting at the same, rectangular table, the experimenter sitting at the long side $(73.5 \mathrm{~cm})$ and the participant at the shorter side $(69 \mathrm{~cm})$ (see Fig. 2). The experimenter was seated to the right of the participant at an angle of $90^{\circ}$ and using her right hand performing the movements. This position provided the participant with a view at the inside of her hand and its movement, at a distance of $60 \mathrm{~cm}$. Each trial started with the experimenter's hand positioned on a marked spot $8 \mathrm{~cm}$ from the edge of the table along her midsagittal axis, all fingertips touching each other. The cube was positioned $27 \mathrm{~cm}$ further from that point on in the same direction. Thus, all movements made by the experimenter covered a distance of $27 \mathrm{~cm}$.

\section{[ FIGURE 2 ]}

\section{Procedure}

At the start of each trial, the participant was asked to position his or her hand on a marked spot $8 \mathrm{~cm}$ from the edge of the table, all fingertips touching each other, similar to the position of the experimenter's hand at the beginning of a trial. The participant was asked to close his or her eyes and to open them again at the presentation of an auditory signal, announcing the beginning of a trial. At the presentation of the auditory signal and subsequent opening of the eyes, the participant was required to grasp a cube (without taking it from its place) placed at a distance of $27 \mathrm{~cm}$ in front of the participant along his or her midsagittal axis. At the presentation of the auditory signal, the experimenter also started her grasping or pointing movement. The participant was asked not to focus on his or her own cube during the execution of the grasping movement but to observe the movement made by the experimenter.

The grasping movement made by the participant was recorded for $2 \mathrm{~s}$ starting at the presentation of the auditory signal, the beginning of a trial. At the end of one trial the participant was asked to return with the right hand to the starting position, close the eyes and wait for the next trial.

Ten instruction trials preceded the two blocks of experimental trials. These ten trials were pseudorandomly selected from the 16 different conditions in this experiment. Four distracter trials and six test trials were included in the instruction.

\section{Statistical analyses}

Only the trials in which the participant grasped the medium cube were included in the analysis. A single group repeated-measures design was used to compare means. The two-way analysis of variance (ANOVA) that was carried out included the within-subjects factors 'observed cube size' (towards which the experimenter made her movements, $n=4$; large cube, medium cube, small cube and no cube) and 'observed movement' (made by the experimenter, $n=2$; grasping and pointing). The main dependent variable was the participant's average MGA for each test condition. In addition, further 
two-way ANOVA's were performed for average grip aperture at 25, 50 and 75\% (GA25, GA50, GA75) of the movement and for the other kinematic variables: MT, PV, TPV, \%TPV, TMGA and \%TMGA. The Student Newman-Keuls test was used for all post hoc comparisons.

\section{RESULTS}

After reading the instructions and performing ten instruction trials, all subjects understood the task. Nevertheless, 82 trials of the 1,344 trials had to be repeated (6.1\%). Most of the errors were due to poor synchrony between the movements of the experimenter and the participant, caused by long latencies or anticipations at the start of the movement by the participant or the experimenter. Less frequently the trials had to be repeated because of technical problems. Unsuccessful trials were rerun at the end of their block. The number of errors made by the subjects individually ranged from 3 to 13 and the errors were randomly distributed among conditions.

During data analysis, one of 960 test trials was considered a missing value $(0.1 \%)$, caused by unnoticed long latency at the start of the movement.

\section{Grip aperture}

The two-way ANOVA with (observed) cube size and movement type as within-subjects factors yielded no significant main effect of cube size on MGA (see Table 2). There was also no significant difference in MGA between the grasping conditions and the pointing conditions. The analysis did reveal a significant interaction between cube size and movement on MGA (see Table 2, Fig. 3). Posthoc comparison (Student Newman-Keuls test) revealed a significant difference in MGA between the observation of grasping the large cube and grasping the medium cube (see Table 3). The difference in MGA between the observation of grasping the large cube and grasping no cube also was significant. MGA during observation of grasping the small cube did not differ from any of the other cube size conditions. Within the pointing conditions, not one significant difference in MGA was found between the different cube sizes (see Table 3).

\section{[ TABLE 2 ] \\ [ FIGURE 3 ] \\ [ TABLE 3 ]}

No main effects of observed movement type and observed cube size were also found for grip aperture at 25, 50 and $75 \%$ of the movement (see Table 2). However, a significant interaction was observed for all three dependent measures. Post hoc Student Newman-Keuls comparisons showed no significant difference between objects sizes for either pointing or grasping at 25 or $50 \%$ of the movement (see Table 3, Fig. 3). At 75\% of the movement a significant difference was found between the grip aperture during observation of grasping a $100 \mathrm{~mm}$ object and a $50 \mathrm{~mm}$ object. The difference between observation of grasping a $100 \mathrm{~mm}$ and grasping no object was also significant. None of the other comparisons were significant.

\section{Other kinematic variables}

ANOVA's were also performed with the other kinematic variables described. A significant main effect of object size was found for MT (see Table 4). Post hoc comparisons revealed that MT was significantly longer when observing movements towards the largest object size $(100 \mathrm{~mm})$ as compared to the smallest object size $(10 \mathrm{~mm}, P<0.01)$ and when no object was present $(P<0.05)$. No significant effect was found for any of the other kinematic variables.

\section{[ TABLE 4 ]}

\section{DISCUSSION}

The current study aimed to investigate whether observation of a grasping movement influences performance of an ongoing grasping movement. Previous studies with monkeys showed that 'mirror neurones' are activated by observation of grasping movements as well as by performing the grasping movements. Although several studies have investigated the effect of movement observation on movement execution in humans (Iacoboni et al. 1999; Kilner et al. 2003), few studies have assessed whether goal-directed grasping movements are influenced by observation of another grasping 
movement (Castiello et al. 2002; Edwards et al. 2003). We asked subjects to perform prehension movements while observing an experimenter performing grasping movements towards objects identical or dissimilar in size. We hypothesized that observing a grasping movement that was dissimilar would cause activation of grasping representations that were different to the ongoing grasping movement and would cause interference.

The present results suggest that the observation of particular grasping movements made by another person has a measurable interference effect on simultaneously executed grasping movements. Results of a repeated-measures two-way ANOVA on MGA revealed no main effects for the experimenter's cube size and movement type. However, the interaction of cube size and movement type was significant. This interaction effect was due to an effect of cube size on subjects MGA in the grasping conditions. The lack of interference effect within the pointing conditions, suggests that observation of an object-oriented hand movement in itself has no effect on MGA, neither did the sole presence of a differently sized object. Apparently, for the observation of an object-oriented hand movement to influence the simultaneous execution of a grasping movement, it is crucial that the observed movement is also a grasping movement. The interference effect, therefore, cannot simply be accounted for by attentional demands or task complexity.

Post-hoc analyses revealed that the results are overall in line with our expectations. We found that subjects' MGA in the congruent condition (e.g. when the objects to be grasped by the experimenter and participant were both $50 \mathrm{~mm}$ ) did not differ from baseline condition in which no object was present. When observing grasping towards the large object, participants opened their hand wider as compared to the baseline condition and the congruent condition.

However, in the incongruent condition when the small object was grasped, MGA was not significantly different compared to the congruent and baseline conditions.

There may be several possible explanations for the lack of an interference effect when the participant observed the prehension of the small object. First, it may related to the fact that we measured maximum grip aperture as principal dependent variable. Any subtle effects of the grasping observation during the course of opening the hand may obscured as the participants need to reach the appropriate MGA in order to grasp the object successfully. However, further analyses of grip aperture at various stages of the movement (25, 50 and $75 \%$ ) also failed to reveal an effect of movement observation for the smallest object, suggesting that this is unlikely to be the case.

A second reason may be the manner in which the small cube was grasped, compared to the medium and large cube. The medium and large cube were grasped with all fingers from above, the small cube was grasped with only the thumb and index finger from above (see Fig. 1). Perhaps the slightly deviant grasp in 'precision grip' differed to such an extent from the 'all fingers prehension' grasp that it caused less interference when performing an 'all fingers prehension' movement. This may be similar to the pointing movements, which also did not affect prehension movements. Possibly, only when the two movements are alike, interference occurs. A final possibility is that the incongruency between the observed and executed prehension resulted in greater uncertainty in the performance of the participant's grasping movement. It is well known that when subjects are uncertain during the grasp they open their hand wider (Paulignan et al. 1991), which could have obscured any effect of observing grasping the small object. Indeed, MGA was larger when observing grasping the small object than when observing the medium object, although this effect was not significant.

The results of this study are consistent with other recent behavioural studies. Kilner et al. (2003) found an interference effect of movement observation on simultaneous movement execution. The effect observed in the current study concerned object-oriented grasping movements, whereas in the Kilner study the movements were of non-goal-directed sinusoidal nature. Our result confirms their idea that the effect they found is due to interference within a common neural network that encodes both observed and executed movements, although our experimental set-up may be more akin to the original mirror neuron studies. Other studies did assess the effect of grasping observation on grasping execution (Castiello et al. 2002; Edwards et al. 2003). Castiello et al. (2002) found a priming effect of the prior observation trial on the kinematics of reaching and grasping a target object and this observation was confirmed by Edwards et al. (2003). There are however several crucial differences with the current study. First, our study showed an on-line interference effect, instead of following a prime. Thus, the priming effect of Castiello et al. (2002) influences the programming of the subject's MGA (but note that Edwards et al. did not find an effect on MGA), whereas the current study showed 
an interference effect on the on-line control of the subject's MGA. The idea that effects of grasping observation in the current study were related to the online control of the hand opening rather than prior programming is supported by a lack of effect on grip aperture earlier in the movement (at 25 and 50\% of movement time). By contrast, at $75 \%$ similar differences were found as for MGA. Secondly, Castiello et al. (2002) investigated the effect of prime validity, rather than the effect of distracter object size. Thus in our study, we observed only an effect when the distracter object size was larger, while Castiello and colleagues assessed whether the prime was valid irrespective of relative object size. Finally, Edwards et al. (2003) also showed priming of the mere presence of a target object and found that several kinematic variables were affected in addition to MGA (MT, TPV, TMGA). In our study only MT was additionally influenced (by object size irrespective of type of observed movement). Overall, our data tentatively suggests a more specific effect with respect to the observed action (only grasping towards a large object) and the type of variable affected (MGA). Of course, online interference may provide less time for any effect grasping observation to occur. Another possible explanation for the differences between the studies may be that different components of the mirror neuron system are involved. Neurophysiological studies indicate that mirror neurons may differ their activation with respect to the course of the movement. Thus some show a higher level of activity during the last part of a grasping movement (Umilta et al. 2001), other continue to fire after the movement has ended (Gallese et al. 1996). In humans, TMS studies show changes in cortico-spinal excitability during grasping observation (Gangitano et al. 2001, 2004). More specifically, the changes in cortico-spinal excitability appear to be modulated by kinematic changes in the observed movement and the peak value was obtained at the time of observed MGA. The observed changes in MGA of the participant in the current study may be behavioural correlate of such neurophysiological changes during grasping observation.

As already mentioned, the only other kinematic variable influenced by observing the experimenter making a visuomotor response was movement time. However, only a main effect of experimenter's object size was found and no main effect of movement type or interaction between movement type and object size. These findings therefore suggest that the size of the object irrespective of the observed movement influenced MT. Therefore this effect cannot be ascribed to activation of the mirror system, but may be related to a general attentional effect of focusing on a large non-target object during the grasping movement.

Overall, this study provides evidence that online interference occurs when an observed grasping movement is of a larger aperture than the grasping movement simultaneously executed. This finding, together with recent TMS studies on cortico-spinal excitability suggests that observing a movement automatically generates an internal representation of that movement. Such an internal representation can cause interference in the execution of the grasping movement, when active at the same time.

This finding is consistent with an accumulating body of evidence for the existence of a human motor vocabulary in which motor representations are activated automatically during the observation of motor actions.

\section{ACKNOWLEDGMENTS}

This work was supported by a Vidi research grant from the Netherlands Organisation for Scientific Research [NWO 452-03-325]. We thank Pien Grootenboer and Ingeborg Smit for their help with the data collection and Rob McIntosh for his assistance with the data analysis.

\section{REFERENCES}

Castiello U, Lusher D, Mari M, Edwards MG, Humphreys GW (2002) Observing a human or a robotic hand grasping an object: differential motor priming effects. In: Prinz W, Hommel B (eds) Attention and performance, vol XIX. MIT Press, Cambridge

Craighero L, Bello A, Fadiga L, Rizzolatti G (2002) Hand action preparation influences the responses to hand pictures. Neuropsychologia 40:492-502

Edwards MG, Humphreys GW, Castiello U (2003) Motor facilitation following action observation: a behavioural study in prehensile action. Brain Cogn 53:495-502

Fadiga L, Fogassi L, Pavesi G, Rizzolatti G (1995) Motor facilitaction during action observation: a magnetic stimulation study. J Neurophysiol 73:2608-2611 
Dijkerman, H.C., Smit, M.C.

Interference of grasping observation during prehension, a behavioural study.

Experimental Brain Research: 2006, nr. 8, p.

Fadiga L, Fogassi L, Gallese V, Rizzolatti G (2000) Visuomotor neurons: ambiguity of the discharge or 'motor' perception? Int J Psychophysiol 35:165-177

Gallese V, Fadiga L, Fogassi L, Rizzolatti G (1996) Action recognition in the premotor cortex. Brain 119:593-609

Gangitano M, Mottaghy FM, Pascual-Leone A (2001) Phase-specific modulation of cortical motor output during movement observation. Neuroreport 12:1489-1492

Gangitano M, Mottaghy FM, Pascual-Leone A (2004) Modulation of premotor mirror neuron activity during observation of unpredictable grasping movements. Eur J Neurosci 20:2193-2202

lacoboni M, Woods RP, Brass M, Bekkering H, Mazziotta JC, Rizzolatti G (1999) Cortical mechanisms of human imitation. Science 286:2526-2528

Jeannerod M, Arbib MA, Rizzolatti G, Sakata H (1995) Grasping objects: the cortical mechanisms of visuomotor transformation. Trends Neurosci 18:314-320

Kilner JM, Paulignan Y, Blakemore SJ (2003) An interference effect of observed biological movement on action. Curr Biol 13:522-525

Koski L, Wohlschlager A, Bekkering H, Woods RP, Dubeau MC, Mazziotta JC, Iacoboni M (2002) Modulation of motor and premotor activity during imitation of target-directed actions. Cereb Cortex 12:847-855

Nishitani N, Hari R (2000) Temporal dynamics of cortical representation for action. Proc Natl Acad Sci USA 97:913-918

Paulignan Y, Jeannerod M, MacKenzie C, Marteniuk R (1991) Selective pertubation of visual input during prehension movements 2. The effects of changing object size. Exp Brain Res 87:407-420

di Pellegrino G, Fadiga L, Fogassi L, Gallese V, Rizzolatti G (1992) Understanding motor events: a neurophysiological study. Exp Brain Res 91:176-180

Rizzolatti G, Arbib MA (1998) Language within our grasp. Trends Neurosci 21:188-194

Rizzolatti G, Fadiga L, Gallese V, Fogassi L (1996a) Premotor cortex and the recognition of motor actions. Cogn Brain Res 3:131-141

Rizzolatti G, Fadiga L, Matelli M, Bettinardi V, Paulesu E, Perani D, Fazio F (1996b) Localization of grasp representations in humans by PET: 1. Observation versus execution. Exp Brain Res 111:246252

Umilta MA, Kohler E, Gallese V, Fogassi L, Fadiga L, Keysers C, Rizzolatti G (2001) I know what you are doing. a neurophysiological study. Neuron 31:155-165

Wohlschlager A, Bekkering $\mathrm{H}$ (2002) Is human imitation based on a mirror-neurone system? Some behavioural evidence. Exp Brain Res 143:335-341

\section{TABLES AND FIGURES}

\begin{tabular}{|c|c|c|c|c|c|}
\hline \multirow{2}{*}{$\begin{array}{l}\text { Table } 1 \text { The experimental } \\
\text { design }\end{array}$} & & \multirow[t]{2}{*}{ Observed action } & \multicolumn{3}{|l|}{ Performed action } \\
\hline & & & $\begin{array}{l}\text { Grasping } 100 \mathrm{~mm} \\
\text { object }\end{array}$ & $\begin{array}{l}\text { Grasping } 50 \mathrm{~mm} \\
\text { object }\end{array}$ & $\begin{array}{l}\text { Grasping } 10 \mathrm{~mm} \\
\text { object }\end{array}$ \\
\hline & Incongruent & Grasping $100 \mathrm{~mm}$ object & 4 Non-analysis & 10 Test & 4 Non-analysis \\
\hline & Congruent & Grasping $50 \mathrm{~mm}$ object & 4 Non-analysis & 10 Test & 4 Non-analysis \\
\hline & Incongruent & Grasping $10 \mathrm{~mm}$ object & 4 Non-analysis & 10 Test & 4 Non-analysis \\
\hline & Baseline & Grasping without object & 4 Non-analysis & 10 Test & 4 Non-analysis \\
\hline & Incongruent & Pointing $100 \mathrm{~mm}$ object & - & 10 Control & - \\
\hline & Congruent & Pointing $50 \mathrm{~mm}$ object & - & 10 Control & - \\
\hline & Incongruent & Pointing $10 \mathrm{~mm}$ object & - & 10 Control & - \\
\hline & Baseline & Pointing without object & - & 10 Control & - \\
\hline & $\begin{array}{l}\text { Total number } \\
\text { of trials }\end{array}$ & & 16 & 80 & 16 \\
\hline
\end{tabular}


Dijkerman, H.C., Smit, M.C.

Interference of grasping observation during prehension, a behavioural study.

Experimental Brain Research: 2006, nr. 8, p.

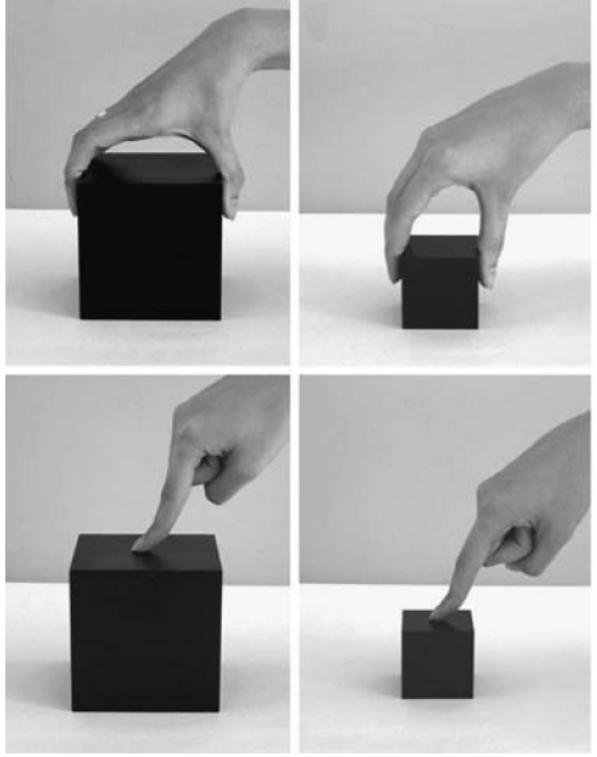

Fig. 1 Top: The grasping stimuli as performed by the experimenter; grasping a large cube, grasping a medium cube, grasping a small cube and grasping without a cube. Bottom: The pointing

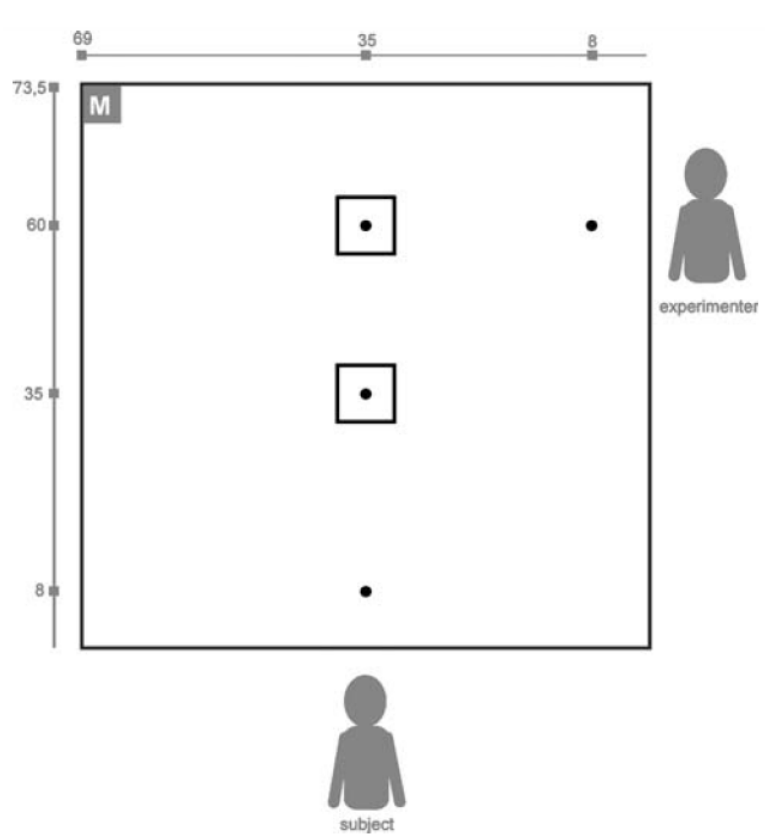

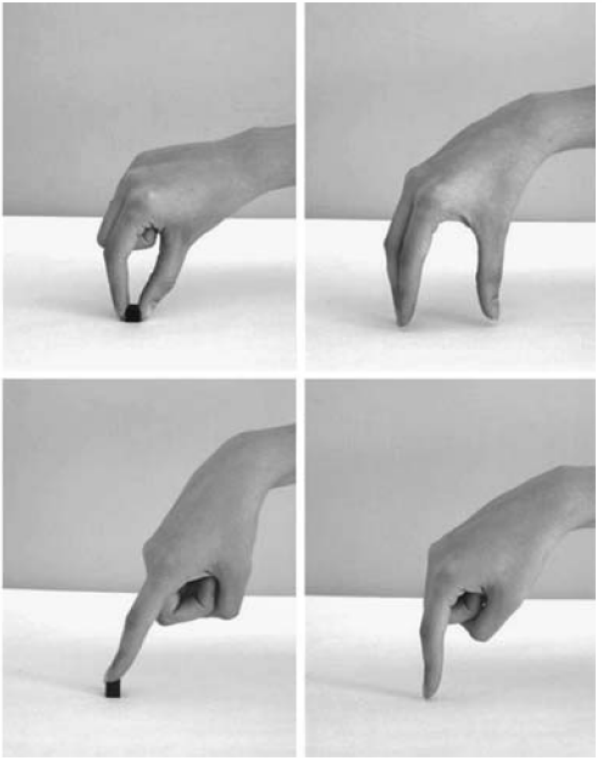

stimuli as performed by the experimenter; pointing at a large cube, pointing at a medium cube, pointing at a small cube and pointing without a cube

Fig. 2 The experimental set-up with the Minibird magnet in the left upper corner

Table 2 The results of a two-way repeated measures ANOVA on grip aperture measures with type of experimenter's movement (grasping or pointing) and experimenter's object size as independent variables

\begin{tabular}{|c|c|c|c|c|}
\hline & MGA & GA25 & GA50 & GA75 \\
\hline $\begin{array}{l}\text { Main effect type } \\
\text { of movement }\end{array}$ & $F(1,11)=0.72, P=0.41$ & $F(1,11)=0.001, P=0.97$ & $F(1,11)=0.50, P=0.49$ & $F(1,11)=0.98, P=0.34$ \\
\hline $\begin{array}{l}\text { Main effect } \\
\text { object size }\end{array}$ & $F(3,33)=2.65, P=0.07$ & $F(3,33)=1.39, P=0.27$ & $F(3,33)=1.34, P=0.28$ & $F(3,33)=1.49, P=0.24$ \\
\hline Interaction & $F(3,33)=5.07 P=0.005^{*}$ & $F(3,33)=3.06, P=0.04 *$ & $F(3,33)=3.15, P=0.04 *$ & $F(3,33)=3.68, P=0.02^{*}$ \\
\hline
\end{tabular}

$M G A$ Maximum grip aperture, GA25 grip aperture at $25 \%$ of the movement, $G A 50$ grip aperture at $50 \%$ of the movement, GA75 grip aperture at $75 \%$ of the movement

*Significant effect 
Dijkerman, H.C., Smit, M.C.

Interference of grasping observation during prehension, a behavioural study.

Experimental Brain Research: 2006, nr. 8, p.

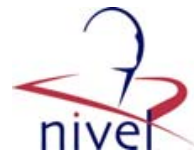

Fig. 3 Mean grip aperture $(+1 \mathrm{SE}$ in $\mathrm{mm})$ during observation of grasping (left in light grey) or pointing (right in dark grey) movements. The top graph shows the maximum grip aperture. The bottom three graphs depict grip aperture at 25,50 and $75 \%$ of the movement respectively. A * denotes a significant difference between conditions. Note that the only those trials in which the participant grasped the medium target object were included in the analysis
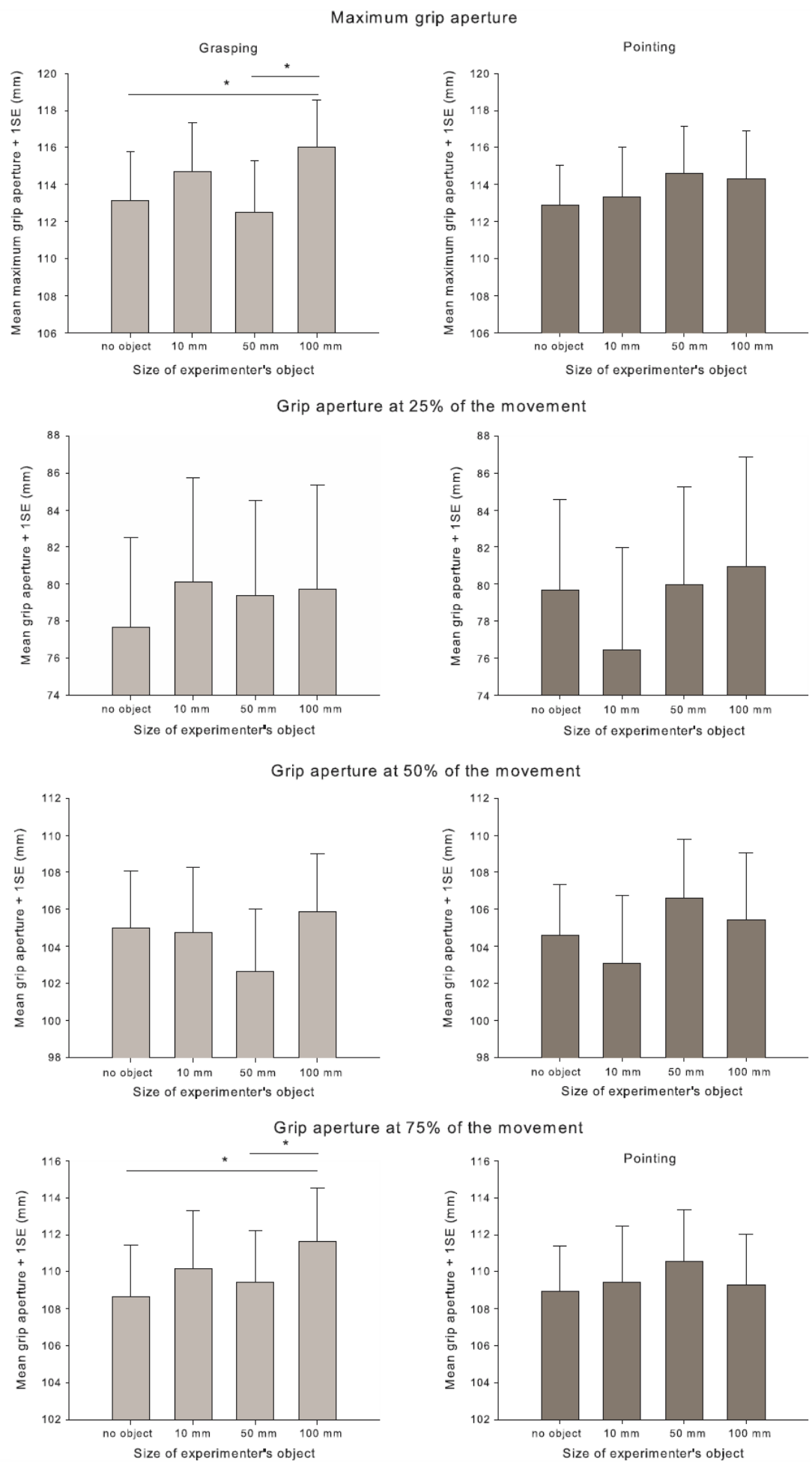
Dijkerman, H.C., Smit, M.C.

Interference of grasping observation during prehension, a behavioural study.

Experimental Brain Research: 2006, nr. 8, p.

Table $3 P$-values for the Student Newman-Keuls post hoc test following the two-way repeated measures ANOVA with maximum grip aperture (MGA), grip aperture at 25, 50 and $75 \%$ of the movement as dependent variables

*Significant difference

\begin{tabular}{|c|c|c|c|c|c|c|}
\hline & \multicolumn{3}{|c|}{ Grasping } & \multicolumn{3}{|c|}{ Pointing } \\
\hline & $\begin{array}{l}10 \mathrm{~mm} \\
\text { object }\end{array}$ & $\begin{array}{l}50 \mathrm{~mm} \\
\text { object }\end{array}$ & $\begin{array}{l}100 \mathrm{~mm} \\
\text { object }\end{array}$ & $\begin{array}{l}10 \mathrm{~mm} \\
\text { object }\end{array}$ & $\begin{array}{l}50 \mathrm{~mm} \\
\text { object }\end{array}$ & $\begin{array}{l}100 \mathrm{~mm} \\
\text { object }\end{array}$ \\
\hline \multicolumn{7}{|l|}{ MGA } \\
\hline No object & 0.27 & 0.69 & $0.0087 \%$ & 0.85 & 0.19 & 0.28 \\
\hline $10 \mathrm{~mm}$ object & & 0.093 & 0.098 & & 0.22 & 0.20 \\
\hline $50 \mathrm{~mm}$ object & & & $0.0017 *$ & & & 0.69 \\
\hline \multicolumn{7}{|l|}{ GA at $25 \%$} \\
\hline No object & 0.55 & 0.26 & 0.52 & 0.14 & 0.98 & 0.90 \\
\hline $10 \mathrm{~mm}$ object & & 0.98 & 0.95 & & 0.19 & 0.067 \\
\hline $50 \mathrm{~mm}$ object & & & 0.97 & & & 0.76 \\
\hline \multicolumn{7}{|l|}{ GA at $50 \%$} \\
\hline No object & 0.85 & 0.45 & 0.81 & 0.95 & 0.83 & 1.0 \\
\hline $10 \mathrm{~mm}$ object & & 0.45 & 0.85 & & 0.21 & 0.66 \\
\hline $50 \mathrm{~mm}$ object & & & 0.27 & & & 0.99 \\
\hline \multicolumn{7}{|l|}{ GA at $75 \%$} \\
\hline No object & 0.41 & 0.87 & $0.013 *$ & 0.82 & 0.31 & 0.66 \\
\hline $10 \mathrm{~mm}$ object & & 0.35 & 0.17 & & 0.45 & 0.88 \\
\hline $50 \mathrm{~mm}$ object & & & $0.04 *$ & & & 0.47 \\
\hline
\end{tabular}

Table 4 Results of the statistical analyses of the kinematic variables other than grip aperture

\begin{tabular}{|c|c|c|c|c|c|c|}
\hline & MT & TMGA & $\%$ TMGA & PV & TPV & $\% \mathrm{TPV}$ \\
\hline $\begin{array}{l}\text { Main effect type } \\
\text { of movement }\end{array}$ & $\begin{array}{c}F(1,11)=0.57 \\
P=0.47\end{array}$ & $\begin{array}{c}F(1,11)=0.38 \\
P=0.55\end{array}$ & $\begin{array}{c}F(1,11)=0.77 \\
P=0.40\end{array}$ & $\begin{array}{c}F(1,11)=0.65 \\
P=0.44\end{array}$ & $\begin{array}{c}F(1,11)=1.31 \\
P=0.28\end{array}$ & $\begin{array}{c}F(1,11)=0.71 \\
P=0.42\end{array}$ \\
\hline $\begin{array}{l}\text { Main effect } \\
\text { object size }\end{array}$ & $\begin{array}{c}F(3,33)=7.0 \\
P=0.001^{*}\end{array}$ & $\begin{array}{c}F(3,33)=1.17 \\
P=0.34\end{array}$ & $\begin{array}{c}F(3,33)=2.27 \\
P=0.10\end{array}$ & $\begin{array}{c}F(3,33)=1.25 \\
P=0.31\end{array}$ & $\begin{array}{l}F(3,33)=0.02 \\
P=1.0\end{array}$ & $\begin{array}{l}F(3,33)=1.18 \\
P=0.33\end{array}$ \\
\hline Interaction & $\begin{array}{c}F(3,33)=0.47 \\
P=0.71\end{array}$ & $\begin{array}{c}F(3,33)=1.15 \\
P=0.35\end{array}$ & $\begin{array}{c}F(3,33)=1.62 \\
P=0.20\end{array}$ & $\begin{array}{c}F(3,33)=0.69 \\
P=0.57\end{array}$ & $\begin{array}{c}F(3,33)=0.07 \\
P=0.98\end{array}$ & $\begin{array}{l}F(3,33)=0.2 \\
P=0.89\end{array}$ \\
\hline $\begin{array}{l}\text { Grasping } 100 \mathrm{~mm} \\
\text { object }\end{array}$ & 852 ms (30.3) & 554 ms (27.9) & $64.3(2.50)$ & $712 \mathrm{~mm} / \mathrm{s}(28.9)$ & $251 \mathrm{~ms}(13.5)$ & $30.0(2.07)$ \\
\hline $\begin{array}{l}\text { Grasping } 50 \mathrm{~mm} \\
\text { object }\end{array}$ & $828(26.7)$ & $565(25.6)$ & $67.2(2.36)$ & $712(26.8)$ & $254(10.8)$ & $31.1(1.46)$ \\
\hline $\begin{array}{l}\text { Grasping } 10 \mathrm{~mm} \\
\text { object }\end{array}$ & $821(26.6)$ & $536(25.6)$ & $64.7(2.83)$ & $719(29.8)$ & $254(12.2)$ & $31.3(1.78)$ \\
\hline $\begin{array}{l}\text { Grasping without } \\
\text { object }\end{array}$ & $824(30.6)$ & $543(29.7)$ & $64.7(2.62)$ & $713(32.9)$ & $252(13.1)$ & $31.2(1.93)$ \\
\hline $\begin{array}{l}\text { Pointing } 100 \mathrm{~mm} \\
\text { object }\end{array}$ & $849(30.8)$ & $543(24.8)$ & $63.4(2.75)$ & $722(31.2)$ & $258(13.2)$ & $30.9(1.98)$ \\
\hline $\begin{array}{l}\text { Pointing } 50 \mathrm{~mm} \\
\text { object }\end{array}$ & $839(27.6)$ & $548(22.2)$ & $64.6(2.06)$ & $701(27.2)$ & $256(14.1)$ & $31.0(2.04)$ \\
\hline $\begin{array}{l}\text { Pointing } 10 \mathrm{~mm} \\
\text { object }\end{array}$ & $820(27.2)$ & $546(26.7)$ & $65.7(2.81)$ & $717(31.0)$ & $258(16.0)$ & $31.9(2.27)$ \\
\hline $\begin{array}{l}\text { Pointing without } \\
\text { object }\end{array}$ & $834(32.2)$ & $545(28.0)$ & $64.7(2.45)$ & $704(27.5)$ & $258(13.2)$ & $31.3(1.78)$ \\
\hline
\end{tabular}

The top three rows display the results of a two-way repeated measures ANOVA with type of experimenter's movement (grasping or pointing) and experimenter's object size as independent variables. The bottom eight rows display the mean and standard errors (between brackets) per condition

$M T$ Movement time, TMGA time to maximum grip aperture, $\% T M G A$ time to maximum grip aperture as percentage of total movement time, $P V$ peak velocity, $T P V$ time to peak velocity, \% TPV time to peak velocity as percentage of total movement time *Significant effect 